\title{
The role of miR-29b in cancer: regulation, function, and signaling
}

This article was published in the following Dove Press journal:

OncoTargets and Therapy

3 March 2015

Number of times this article has been viewed

\section{Bin Yan ${ }^{1, *}$ \\ Qiong Guo ${ }^{2, *}$ \\ Fa-jun $\mathrm{Fu}^{3}$ \\ Zhao Wang' \\ Zhuo Yin' \\ Yong-bao Wei' \\ Jin-rui Yang'}

'Department of Urology, The Second Xiangya Hospital, Central South University, Changsha, People's

Republic of China; ${ }^{2}$ Department of Urology, The Central Hospital of

Changsha, Changsha, People's Republic of China; ${ }^{3}$ Department of Urology,

The Central Hospital of Changsha,

Changsha, People's Republic of China

*These authors contributed equally to this work
Correspondence: Jin-rui Yang Department of Urology, The Second Xiangya Hospital, Central South University, Changsha 4100II,

People's Republic of China

Email yangjr06@163.com

Fa-jun Fu

Department of Urology, The Central Hospital of Changsha, Changsha, 4I0004, People's Republic of China Email675390797@qq.com
Abstract: MicroRNAs (miRNAs) are endogenous small non-coding RNAs with the capacity to regulate gene expression post-transcriptionally. The miRNA-29 family consists of miR-29a, miR-29b, and miR-29c, among which miR-29b is the most highly expressed and is found at two genomic loci. Recently, numerous studies have demonstrated that aberrant expression of miR$29 \mathrm{~b}$ is common in the majority of human cancers. miR-29b is known to critically affect cancer progression by functioning as a tumor suppressor. However, it may also act as an oncogene under certain conditions. In this review, we illustrate the role of miR-29b in cancer regulation, function, and signaling. This is the first review highlighting the role of miR-29b in cancer. Our review aims to summarize the effects of miR-29b on cancer activity and its interactions with target genes and signaling pathways, as well as to provide therapeutic implications for overcoming cancer chemoresistance.

Keywords: miR-29b, cancer, biological function, oncogene, tumor promoter, chemoresistance

\section{Introduction}

MicroRNAs (miRNAs) are endogenous, small, non-coding RNAs approximately 22 nucleotides in length with the capacity to interfere in the expression of protein-coding messenger RNAs (mRNAs). They work as post-transcriptional regulators of gene expression by binding to the $3^{\prime}$-untranslated region ( $3^{\prime}$-UTR) of target mRNAs. ${ }^{1,2}$ Because of their short sequence, these RNA molecules are able to mediate mRNA degradation by perfect base-pairing with target mRNAs or by translational blockade via imperfect base-pair binding to a wide range of target genes. One miRNA might bind to a number of mRNA transcripts, and in turn, one mRNA can be targeted by a widespread panel of miRNA species. ${ }^{1}$ Consequently, miRNAs can dictate cell fate, cell cycle regulation, as well as cell proliferation, differentiation, apoptosis, and invasion. ${ }^{3}$

Cancer is one of the leading causes of death worldwide. Accumulating evidence has demonstrated that miRNAs act as crucial regulators of cancer by targeting different signaling pathways and multiple mRNA transcripts. Moreover, a strong association of miRNA expression profiles with the etiology, classification, progression, and prognosis of multiple human cancers has been established. miRNAs can act as either oncogenes or tumor suppressors. ${ }^{4,5}$

The miR-29 family consists of miR-29a, miR-29b, and miR-29c, differing only in two or three bases. miR-29b includes two sub-family members, miR-29b1 and miR-29b2. The miR-29 family is encoded and transcribed in tandem by two genes located on chromosome 7q32.3 (miR-29a/b1 locus) or chromosome1q32.2 (miR-29a/ b2 locus), respectively. Mature miR-29b is therefore encoded by two distinct precursor 
stem sequences (pre-miRNA) on both chromosomes, a pre-miR-29b1 and pre-miR-29b2 stem. Although the sequences of the two pre-miR-29b stems are different, the mature miR-29bs resulting from these two stem structures are identical. ${ }^{6} \mathrm{miR}-29 \mathrm{~b}$ shares gene regulatory function with other members of the miR-29 family. Expression of all miR-29 family members is abnormal in malignant cells, and they all exert anti-tumor function. Moreover, overlapping functions of miR-29 family members include transcriptional regulation, epigenetic modulation, and cell apoptosis. However, despite the high similarity of mature $\mathrm{miR}-29 \mathrm{a} / \mathrm{b} / \mathrm{c}$ sequences, numerous studies have showed that different isoforms of the miR-29 family may exert distinct functions. ${ }^{7,8}$ For instance, enhancer-binding protein-alpha (CEBPA) selectively regulates miR-29b expression at its miR-29a/b1 locus on chromosome 7q32.3, whereas miR$29 \mathrm{~b} 2 /$ c on chromosome 1q32.2 is not affected. ${ }^{9}$

Recently, overwhelming evidence has suggested that aberrant expression of the members of the miR-29 family is involved in multiple cancers. As a member of the miR-29 family, miR-29b is generally recognized as a fundamental regulator of epithelial-mesenchymal transition (EMT), a pathway involved in cancer metastasis and chemoresistance. As miR-29b is the most highly expressed miR-29b family member and is found at two genomic loci, this review will focus on the association between miR-29b dysregulation and cancer.

\section{miR-29b and its regulation}

Recent reports have shown that miR-29b is ectopically expressed in malignant cancer. Several genes regulating miR-29b expression have been identified. C-Myc is known to directly suppress miR-29s. ${ }^{10}$ In addition, Zhang et al showed that miR-29 is repressed by MYC through cooperative regulation by histone deacetylase 3 and histone-lysine $\mathrm{N}$-methyltransferase 2 (EZH2) in aggressive B-cell lymphomas (BCLs). MYC upregulates EZH2 via repression of the EZH2-targeting miR-26a, and EZH2 induces MYC via inhibition of the MYC-targeting miR-494, thereby creating a positive feedback loop and increasing the expression of MYC. ${ }^{11}$ Consistent with this finding, HDACs are overexpressed in chronic lymphocytic leukemia (CLL), thus silencing the expression of critical tumor suppressors miR-29b, miR-15a, and miR-16. ${ }^{12}$

In addition to c-Myc, miR-29 expression is also suppressed by hedgehog, nuclear factor kappa-light-chainenhancer of activated B cells (NF- $\mathrm{KB})$, and transforming growth factor beta (TGF- $\beta$ ) signaling since two putative E-box (Myc-binding) sites, a Gli-binding site, and four
NF-кB-binding sites were identified in the human miR-29a/ b1 promoter sequence. ${ }^{13-15}$ In addition, Jonathan et al identified that the miR-29a/b1 promoter contains three GATA3binding sites, and that GATA3 may inhibit TGF- $\beta$ and NF- $\kappa$ B pathways. Thus, miR-29b expression is induced by GATA3 directly (via binding to the GATA sites in the promoter) or indirectly (by inhibiting TGF- $\beta$ and NF- $\kappa \mathrm{B}$ signaling). ${ }^{16}$

MBP-1, a transcriptional co-repressor called the c-Myc promoter binding protein, may induce a five to nine-fold upregulation of miR-29b in prostate cancer cells, which in turn exerts tumor suppressor effects. ${ }^{17}$ Somatic cell reprogramming factor Sox 2 directly regulates endogenous miR-29b expression and is significantly involved in the regulation of mesenchymal-to-epithelial transition. ${ }^{17}$ Meanwhile, the female hormone progesterone $\mathrm{P} 4$ rapidly downregulates the expression of members of the miR-29 family, particularly in CD44(+) cell populations, thereby potentiating the expansion of $\mathrm{CK5}(+)$ and CD44(+) cells in response to progestins. These findings may illustrate a new mechanism in the progression of hormone-responsive breast cancers. ${ }^{18}$ In melanoma, the miR-29a/b1 cluster was upregulated by interferon- $\gamma$ in a signal transducer and activator of transcription 1 (STAT1)-dependent way, adding a novel regulatory loop in interferon- $\boldsymbol{\gamma}$-mediated Jak/STAT signaling in melanoma cells. ${ }^{19}$

In addition, CCAAT/enhancer-binding protein-alpha (CEBPA), a crucial factor for normal granulopoiesis that is frequently disrupted in acute myeloid leukemia (AML), was found to directly activate miR-29a/b1 expression. ${ }^{9}$ Moreover, two putative responsive elements of the myeloid master regulator PU.1 (SPI1) were identified in the miR-29b2/ miR-29c gene. Further studies showed that PU.1 and MYC transcriptionally downregulated the expression at the miR29 b2/c locus in acute promyelocytic leukemia cells. ${ }^{20}$

\section{Function of miR-29b as a tumor suppressor \\ Role of miR-29b in epigenetic regulation}

The term "epi-miRNAs" has been proposed to indicate a specific class of tumor suppressor miRNAs that are able to downregulate epigenetic enzymes, which, in turn, negatively affect the expression of tumor suppressor genes. Epigenetic silencing of tumor suppressor genes frequently occurs, and this may account for their inactivation in cancer cells. Recently, miR-29b was proved to be an epi-miRNA that targets DNA methyltransferases (DNMTs) and/or regulates DNA demethylation pathway members, leading to the downregulation of global DNA methylation in malignant cells. 
Furthermore, it can regulate the balance of DNA methylation and demethylation by repressing the activities of DNMTs and DNA demethylases.

In AML cells, forced expression of miR-29b resulted in marked reduction of the expression of DNMT1, DNMT3A, and DNMT3B at both RNA and protein levels by direct interaction. miR-29b downregulates DNMT1 indirectly by targeting Sp1, a transactivator of the DNMT1 gene. This in turn leads to a decrease in global DNA methylation and re-expression of p15 (INK4b) and ESR1, two tumor suppressors. ${ }^{21}$ Moreover, miR-29 family members may exert demethylation effects through recurrently regulating the active DNA demethylation pathway members tet methylcytosine dioxygenase (TET1) and thymine DNA glycosylase (TDG). ${ }^{22}$

In multiple myeloma (MM) cells, the miR-29b target mRNAs, DNMT3A and DNMT3, reduce global DNA methylation. Amodio et al identified that transfection of MM cells with synthetic miR-29b mimics the impairment of cell cycle progression and potentiates the growth-inhibitory effects of demethylating agent 5-azacitidine significantly. ${ }^{23}$ miR-29b is also involved in cancer-mediated inflammatory responses. Interleukin-15, a single pro-inflammatory cytokine, causes chromosomal instability and DNA hypermethylation via miR-29b repression, leading to aggressive, acute leukemia of large granular lymphocytes. Drug targeting that reverses miR-29b repression cures the otherwise fatal large granular lymphocytic leukemia. ${ }^{24}$

Suppressor of cytokine signaling-1 (SOCS-1) is a hypermethylated tumor suppressor gene. Amodio et al demonstrated that miR-29b induces SOCS-1 expression by promoter demethylation and, consequently, negatively regulates the migration of $\mathrm{MM}$ and endothelial cells in $\mathrm{MM}$ cells. miR-29b also mediates previously unknown epigenetic functions of the proteasome inhibitor bortezomib in MM cells, as induction of miR-29b by the proteasome bortezomib could mimic the effects induced by forced expression of synthetic miR-29b in MM cells. ${ }^{25}$ Interestingly, bortezomib exerted a dose-dependent increase in miR-29b expression in AML blasts, followed by decreased DNMTs, reduced proliferation, and increased apoptosis. A study by Bernot et al showed that liposomal-encapsulated bortezomib, as a single agent, had higher activity against dKI AML than normal bortezomib. ${ }^{26}$ These studies suggest that liposomal bortezomib may represent a powerful and potentially curative approach for high-risk human diseases. ${ }^{25,26}$

In lung cancer, it has also been demonstrated that DNMTs are targets of miRNA-29b. ${ }^{27,28}$ In DU145 and TRAMP-C1 prostate and MDA-MB-231 breast cancer cells, activation of toll-like receptor 3 (TLR3) induces miRNA expression, including DNMT-targeting miR-29b, resulting in demethylation and re-expression of the oncosuppressor retinoic acid receptor beta. This provides evidence of how miR-29b may be involved in the antitumoral effects of TLR3 through epigenetic modulation. ${ }^{29}$

Interestingly, while miR-29b directly targets DNMTs, a recent study has demonstrated that it may also repress the activity of DNA demethylases, which have opposing roles in controlling the status of DNA methylation. DNMTs and two major factors involved in DNA demethylation, TET1 and TDG, were inhibited directly by miR-29 family members. In this regard, miR-29 upregulated DNA methylation in lung cancer PC9 cells but downregulated methylation in lung cancer A549 cells. This suggested that miR-29 may act as a stabilizer of DNA methylation by protecting against changes in the existing DNA methylation status, thereby suppressing tumorigenesis. These studies provide insights whereby miR-29b, along with other epi-miRNAs, exerts epigenetic effects in multiple ways, rather than by simply preventing DNA methylation. However, further research is needed to elucidate the precise mechanisms of action. ${ }^{30}$

\section{Role of miR-29b in cell proliferation, apoptosis, and differentiation}

Myeloid cell leukemia-1 (Mcl-1) protein, a potent, multidomain anti-apoptotic protein of the B-cell lymphoma (Bcl-2) family, is downregulated by miR-29b. miR-29b was downregulated in malignant $\mathrm{KMCH}$ cholangiocarcinoma (CCA) cells, concomitant with Mcl-1 protein upregulation. Furthermore, forced miR-29b expression in $\mathrm{KMCH}$ cells reduced Mcl-1 protein expression and sensitized the cancer cells to tumor necrosis factor-related apoptosis-inducing ligand cytotoxicity, indicating that miR-29b is a regulator of Mcl-1 protein expression, and thereby regulates apoptosis. ${ }^{31}$ In glioblastoma multiforme-initiating cells, functional studies also showed that miR-29a and miR-29b targeted Mcl-1 mRNA, increasing apoptosis. ${ }^{32}$ In AML cell lines and primary samples, restoration of miR-29b induces apoptosis and significantly reduces tumorigenicity. Transcriptome analysis after ectopic transfection of synthetic miR-29b into leukemia cells indicates that miR-29b targets apoptotic, cell cycle, and proliferation pathways. ${ }^{33}$ In addition, miR-29 may sensitize hepatocellular carcinoma (HCC) cells to apoptosis by directly targeting the anti-apoptotic molecules Bcl-2 and Mcl-1. Moreover, the ability of HCC cells to form tumors in nude mice was dramatically repressed by the introduction of miR-29. These results suggested that apoptosis may be 
promoted by miR-29 via a mitochondrial pathway involving Mcl-1 and Bcl-2. ${ }^{34}$ In human myeloma cell lines, forced miR-29b expression by using the target miRNA-29b expression vector (Ad5F11p-miR-29b) may induce apoptosis and elevate caspase- 3 activation. ${ }^{35}$

Meanwhile, p53, a tumor suppressor that induces apoptosis in many tumor types, is positively regulated by miR-29b. Park et al found that miR-29 family members (miR-29a, miR-29b, and miR-29c) upregulate p53 levels and induce apoptosis in a p53-dependent manner. Unlike in the direct targeting of p53, miR-29 family members also indirectly target $\mathrm{p} 53$ by suppressing $\mathrm{p} 85$ alpha (the regulatory subunit of PI3 kinase) and cell division cycle 42 (CDC42, a Rho family GTPase), both of which negatively regulate $\mathrm{p} 53$. This work enhanced our understanding of the involvement of miRNA in the p53 pathway. ${ }^{36}$

In AML cell lines, overexpression of miR-29a, miR-29b, or miR-2c markedly inhibited cell proliferation and promoted cell apoptosis by targeting protein kinase B2 (AKT2) and cyclin D2 (CCND2) mRNAs, two key signaling molecules. In a mouse AML model, intravenous injection of each miR-29 family member also significantly relieved leukemic symptoms. ${ }^{37}$ The balance of osteoclast (OCL)-mediated bone resorption and osteoblast (OBL)-dependent bone formation is disrupted in MM cells, which impair OBL function and stimulate OCLs to generate lytic lesions. A recent report by Rossi et al indicated that miR-29b negatively regulates human osteoclastic cell differentiation and function, and it showed that forced expression of miR-29b impairs OCL differentiation and overcomes OCL activation triggered by MM cells, indicating that miR-29b-based treatment may be useful for treating MM-related bone disease. ${ }^{38}$ Taken together, the results indicate that miR-29 plays a role in cell proliferation, apoptosis, and differentiation of malignant cancer by targeting related genes (Figure 1).

\section{The role of miR-29b in cancer cell metastasis and invasion}

Cancer metastasis is a multi-stage process that includes extracellular matrix remodeling, blood vessel recruitment, tumor cell entry and exit from circulation, and survival in distant organs. In addition to its roles in cell proliferation, apoptosis, and differentiation, miR-29b also exerts effects on cell migration and invasion (Figure 1).

Increasing evidence indicates that the tumor microenvironment is an important contributor to malignant tumor progression and metastasis. GATA3 is a transcription factor and a member of a class of pro-differentiation factors modifying the tumor microenvironment. A study by Jonathan et al showed that GATA3 suppresses metastasis

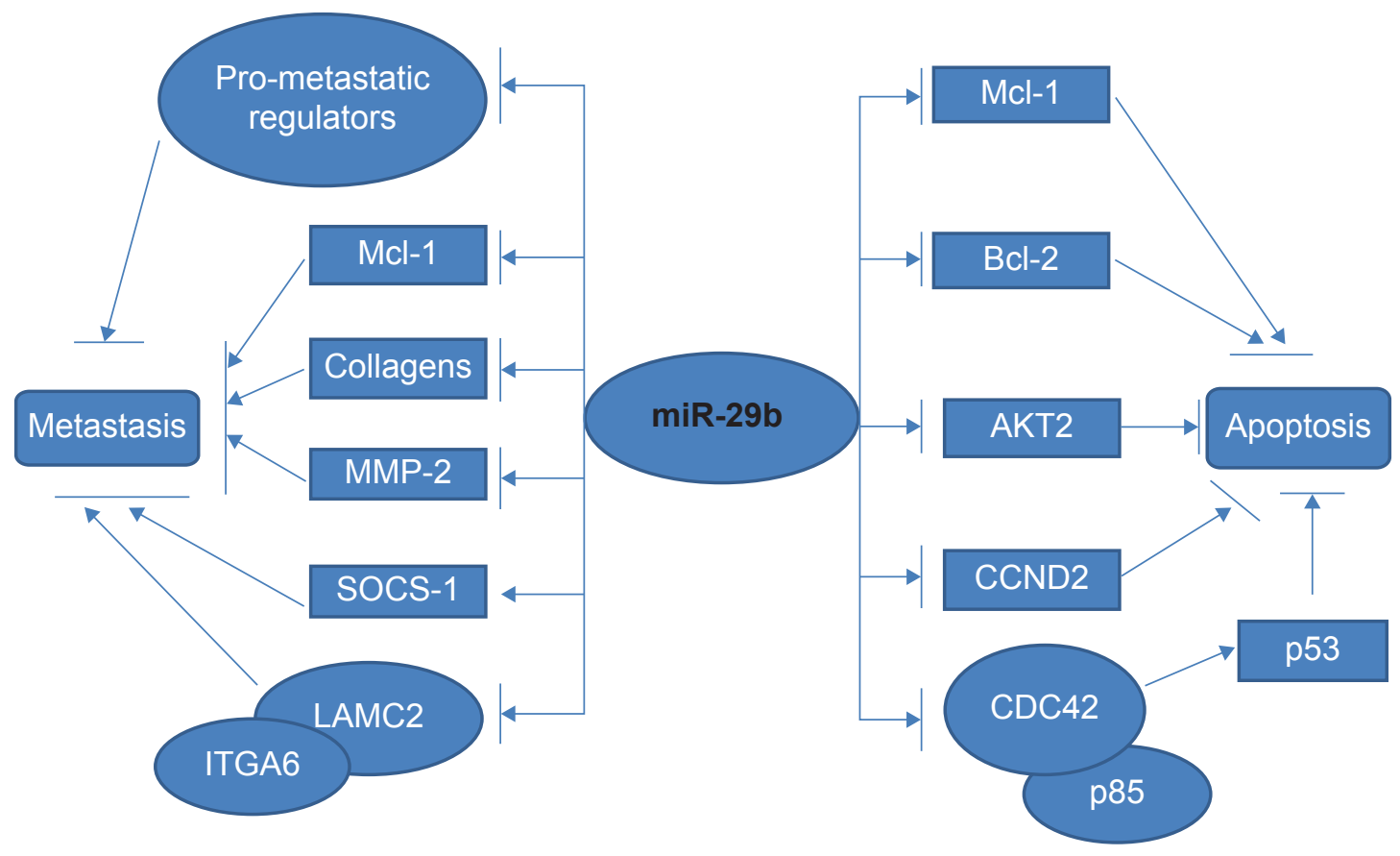

Figure I The mechanism by which miR-29b induces apoptosis and inhibits metastasis in human cancer cells.

Abbreviations: Mcl-I, myeloid cell leukemia-I; MMP-2, matrix metalloproteinase-2; SOCS-I, suppressor of cytokine signaling-I; LAMC2, laminin $\gamma 2$; ITGA6, $\alpha 6$ integrin; miR-29b, micro-ribonucleic acid-29b; Bcl-2, B-cell lymphoma-2; AKT2, protein kinase B; CCND2, cyclin D2; CDC42, cell division cycle 42; PI3, a kinase; p85, the regulatory subunit of PI3 kinase; 5 53, a tumor suppressor that induces apoptosis in many tumor types. 
and alters the tumor microenvironment in breast cancer by inducing miR-29b expression. Mechanistically, miR-29b targets a network of pro-metastatic regulators involved in angiogenesis, collagen remodeling, and proteolysis, including vascular endothelial growth factor alpha (VEGFA), angiopoietin-like 4 (ANGPT-L4), platelet-derived growth factor (PDGF), lipoxygenase (LOX), and matrix metalloproteinase 9 (MMP-9), as well as integrin (ITG) A6, ITGB1, and TGF- $\beta$, thereby inhibiting metastasis. This study sheds light on the network of transcription factors, miRNAs, and the tumor microenvironment to control cancer metastasis. ${ }^{16,39}$

It is well known that EMT is a critical step in tumor cell invasion and metastasis, and it correlates positively with poor patient prognosis. Moreover, it has been shown that miR$29 \mathrm{~b}$ acts as an anti-metastatic miRNA for prostate cancer cells at multiple steps in a metastatic cascade. miR-29b is involved in the inhibition of c-Myc promoter binding protein (MBP-1) in prostate cancer cells through targeting Mcl-1, COL1A1, COL4A1, and MMP-2, which are considered as pro-metastatic matrix molecules implicated in invasion and metastasis. ${ }^{17}$ Reintroduction of miR-29b into metastatic prostate cancer cells inhibits their colonization in the lungs and inhibits metastasis-related traits in vitro by regulating EMT signaling. In one study, epithelial cell marker and E-cadherin expression were enhanced, and N-cadherin, Twist, and Snail expression was downregulated. ${ }^{40}$ In colon cancer cells, Poudyal et al demonstrated that a hexane extract in American Ginseng (HAG) suppressed the migration of colon cancer cells in which miR-29b played a key role. Enhanced HAGinduced expression of miR-29b leads to the migration of colon cancer cells via targeting MMP-2. ${ }^{41}$

Since metastasis is a multi-stage process, epigenetic changes might also play an important role by reducing genome stability. As we mentioned above, miR-29b exerts important epigenetic activity in cancer. miR-29b leads to promoter demethylation of SOCS-1, a hypermethylated tumor suppressor gene, thereby negatively regulating migration of MM and endothelial cells. ${ }^{25}$ Furthermore, a head and neck squamous cell carcinoma (HNSCC) study by Kinoshita et al showed that miR-29s inhibit cancer cell migration and invasion via silencing OF laminin $\gamma 2$ (LAMC2) and $\alpha 6$ ITG (ITGA6) genes. $^{42}$

\section{miR-29b and cancer chemosensitivity}

Chemoresistance to standard anticancer agents is a major problem in almost all cancer therapies. Despite significant improvements in new chemotherapeutic agents, numerous cancer patients ultimately die because of the lack of chemotherapeutic options that are unaffected by chemoresistance. Discovering a strategy to reverse the chemoresistance in cancer is urgently needed. ${ }^{43}$ Since the association of miRNA expression and cancer chemosensitivity was first raised in CCA, many studies have indicated that miRNAs are involved in cancer chemosensitivity. ${ }^{44}$ Meanwhile, accumulating evidence has revealed the vital role of miR-29b in cancer chemosensitivity.

CCA is highly resistant to chemotherapy, including gemcitabine (Gem) treatment. Okamoto et al demonstrated that miR-29b was downregulated in the more Gem-resistant CCA cell line, and that ectopic overexpression of miR-29b enhanced chemosensitivity to Gem CCA cell line HuH28. In addition, two miR-29b target genes, phosphoinositide3-kinase regulatory subunit 1 (PIK3R1) and MMP-2, are responsible for the resistance of CCA Gem treatment. ${ }^{45}$

In ovarian cancer, mouse mucin-1 (MUC1) aptamermiR-29b chimera (Chi-29b) can efficiently inhibit ovarian tumor growth through reducing phosphatase and tensin homologue (PTEN) methylation and inhibiting miR29b-targeted oncogene expression. In accordance with this, intraperitoneal injection of Chi-29b significantly increased apoptosis in paclitaxel-resistant OVCAR-3 cells, suggesting the anti-chemoresistant role of miR-29b in ovarian cancer. ${ }^{46}$ In MM, miR-29b sensitizes MM cells to bortezomib-induced apoptosis through the activation of a feedback loop with the transcription factor $\mathrm{Sp} 1$, which is a transcription factor endowed with oncogenic activity. ${ }^{47}$

In addition, Mims et al demonstrated that an increase of miR-29b expression by AR-42, a potent histone deacetylase inhibitor in AML, improved the anti-leukemia activity of decitabine. One possible explanation for the better activity may relate to miR-29b targeting DNMT expression, thereby resulting in improved treatment response. This provides the hint as to how the epigenetic effects of miR-29b contribute to its chemosensitivity role in cancer. ${ }^{48}$

On one hand, miR-29b could improve chemosensitivity in several cancers. On the other hand, its expression could also be validated as a predictive factor for the clinical response to chemotherapy. miR-29b expression is associated with clinical response to chemotherapy. Two studies by Blum et al identified that subjects who responded to decitabine treatment had a higher pretreatment level of miR-29b than non-responders in older AML patients. In this way, levels of miR-29b could be taken as a predictive factor for stratification to decitabine treatment. ${ }^{49,50}$

While miR-29b is responsible for enhancing cancer sensitivity, free synthetic miRNAs are easily degraded in 
biofluids and have limited cellular uptake. Higher miR-29b pretreatment expression is associated with better response and outcome. Thus, designing a strategy to increase miR-29b levels is necessary. A novel transferrin-conjugated nanoparticle delivery system for synthetic miR-29b (Tf-NP-miR-29b) was proved to be more efficient in targeting oncogenes and in increasing the therapy effects of decitabine. ${ }^{51}$ In addition, $\mathrm{Wu}$ et al developed a cationic lipoplex-based carrier that efficiently delivered miR-29b, showing great potential in the development of miRNA-based therapeutics for lung cancer treatment. ${ }^{52}$

In summary, evidence suggests that miR-29b is a critical regulator of cancer chemosensitivity, mostly through targeting oncogenes, exerting epigenetic regulation, and apoptosis. The studies discussed in this review provide a strong rationale for the possibility of miRNA-related diagnostic, prognostic, and therapeutic strategies to overcome cancer chemoresistance. A better understanding of the mechanisms of miRNA-mediated cancer chemoresistance and an efficient miR-29b delivery strategy would provide a promising new therapeutic strategy for cancer.

\section{The function of miR-29b as a tumor promoter}

As mentioned above, miR-29b is well known for its role as a tumor suppressor. However, many studies have demonstrated that an miRNA may be considered a tumor suppressor or a tumor promoter, depending on its targets in different tissues and cell types. ${ }^{53}$ Controversial issues surrounding the role of miRNA expression have existed for a long time. For example, some studies found that miR-29a was downregulated in AML patients. ${ }^{33}$ However, Han et al reported that miR-29a was overexpressed in AML patients, and that miR-29a could initiate AML. ${ }^{7}$ In addition, Santanam et al reported that the expression of miR-29 family members was higher in indolent human B-cell CLL (B-CLL) than in aggressive B-CLL and normal CD19+ B cells. ${ }^{54}$ Kong et al demonstrated that miR-29a was upregulated in HCC induced by the hepatitis $\mathrm{B}$ virus $\mathrm{X}$ protein and promoted migration by targeting PTEN. ${ }^{55}$ In fact, emerging studies showed that miR-29b may also exert oncogenic effects under specific conditions.

A study by Cheng et al demonstrated the oncogenic potential of miR-29b. This study showed that miR-29 targets the TET2 gene, which is a key tumor suppressor frequently mutated in hematopoietic malignancies. miR-29b, along with miR-125b, miR-29c, miR-101, and miR-7, is preferentially overexpressed in TET2-wild-type AML. It will be interesting to elucidate the underlying mechanism of this effect. ${ }^{56}$ In addition, miR-29b1 and miR-29c levels were higher in the bladder cancer cell line, T24, than in normal urothelial cells. Knockdown of miR-29b1 or miR-29c suppressed T24 cell growth, suggesting an oncogenic role for miR-29b. ${ }^{57}$

In breast cancer cells, a study showed that miR-29b expression was higher in metastatic breast cancer cells and tissues than in low-metastatic breast cancer cells and tissues. Enhanced miR-29b expression promoted cell migration, invasion, and apoptotic resistance through direct repression of PTEN expression. In addition, miR-29b over-expression was correlated with an advanced tumor stage, lymph node metastasis, and poor prognosis, indicating that miR-29b may be useful as a molecular prognostic marker. ${ }^{58}$ Wang et al demonstrated that $\mathrm{Clq}$ and tumor necrosis factor related protein 6 (C1QTNF6), SPARC, and COL4A2 are directly targeted by miR-29b, and downregulation of their mRNAs contributed to the invasiveness of the human breast cancer cell line MCF-7.59 These studies draw the opposite conclusion to a study by Chou et al where miR-29b was demonstrated to inhibit metastasis in breast cancer. ${ }^{16}$

The mechanism for the opposing roles of miR-29b is still unknown. It may be attributed to different sample sizes, genetic backgrounds, clinical characteristics, or different technique platforms. ${ }^{60}$ Further study is needed to elucidate the underlying mechanism.

\section{Gene targets of miR-29b}

It has been established that miRNAs post-transcriptionally mediate mRNA degradation via perfect or imperfect base pairing. There is a wide range of mRNAs that an individual miRNA may target. Since its discovery, miR-29b has been found to target numerous genes and interact with several pathways (Table 1).

In various human cancer cells, miR-29b was reported to be a major mediator of EMT. First, miR-29b negatively regulates the expression of multiple genes involved in regulating the extracellular matrix, including collagens (COL1A1, COL1A2, COL4A1, COL5A1, COL5A2, and COL3A1), LAMC1, and fibrillin (FBN) as well as several genes involved in extracellular matrix deposition and remodeling. Three additional genes, BMP1, ADAM12, and NKIRAS2, were identified as direct targets of miR-29b. ${ }^{61}$ In prostate cancer cells, exogenous expression of miR-29b inhibited Mcl-1, COL1A1, COL4A1, and MMP-2. ${ }^{17}$ Subsequent studies showed that miR-29b directly targets Snail, 
Table I Molecular targets of miR-29b

\begin{tabular}{|c|c|c|c|}
\hline Target molecules & Cellular system & Biological functions & References \\
\hline Collagens, LAMCI, and FBN & Human trabecular meshwork cells & $\begin{array}{l}\text { Synthesis and deposition of extracellular } \\
\text { matrix (ECM) }\end{array}$ & Luna et $a^{61}$ \\
\hline Mcl-I, COLIAI, and COL4AI & Prostate cancer cells & Cancer cell invasion and metastasis & Steele et $\mathrm{al}^{17}$ \\
\hline E-cadherin, N-cadherin, Twist, and Snail & Prostate cancer cells & Epithelial-mesenchymal transition & Ru et $\mathrm{al}^{40}$ \\
\hline $\begin{array}{l}\text { VEGFA, ANGPT-L4, PDGF, LOX, and MMP-9, } \\
\text { and targeting ITGA6, ITGBI, and TGF- } \beta\end{array}$ & Breast cancer cells & $\begin{array}{l}\text { Differentiation, metastasis, and tumor } \\
\text { microenvironment }\end{array}$ & Chou et $\mathrm{al}^{16}$ \\
\hline MCL-I & Acute myelogenous leukemia & $\begin{array}{l}\text { Apoptosis, cell cycle, and proliferation } \\
\text { pathways }\end{array}$ & Garzon et $\mathrm{al}^{33}$ \\
\hline DNMT3A and DNMT3B & Multiple myeloma & Epigenetic modulation & Amodio et $\mathrm{al}^{23}$ \\
\hline PDPN & Glioblastoma & Invasion, apoptosis, and proliferation & Cortez et al ${ }^{65}$ \\
\hline PTEN & Non-small cell lung cancer & Cell apoptosis, and epigenetic modulation & Li et $\mathrm{al}^{66}$ \\
\hline CIQTNF6, SPARC, and COL4A2 & MCF-7 human breast cancer cells & Invasion & Wang et $\mathrm{al}^{65}$ \\
\hline
\end{tabular}

Notes: This table lists the targets of miR-29b and their corresponding biological functions.

Abbreviations: miR-29b, micro-ribonucleic acid-29b; LAMCI, laminin $\gamma \mathrm{l}$; FBN, fibrillin; Mcl-I, myeloid cell leukemia-I; COL, collagen molecular target type; VEGFA, vascular endothelial growth factor $\alpha$; ANGPT-L4, angiopoietin-like 4; PDGF, platelet-derived growth factor; LOX, lipoxygenase; MMP-9, matrix metalloproteinase-9; ITGA6, $\alpha 6$ integrin; ITGAI, $\alpha 2$ integrin; TGF- $\beta$, transforming growth factor $\beta$; DNMT, deoxyribonucleic acid methyltransferase; PDPN, podoplanin; PTEN, phosphatase and tensin homologue; CIQTNF6, CIq and tumor necrosis factor related protein 6; SPARC, secreted protein, acidic and rich in cysteine.

a key marker of mesenchymal cells. ${ }^{40}$ In breast cancer, miR-29b targets a network of micro-environmental genes involved in angiogenesis, collagen remodeling, and matrix degradation, including VEGFA, ANGPT-L4, PDGF, LOX, and MMP-9. Furthermore, it targets ITGA6, ITGB1, and TGF- $\beta$, thereby indirectly affecting differentiation and epithelial plasticity. ${ }^{16}$

Meanwhile, miR-29b exerts tumor suppressive effects by targeting a variety of oncogenes and upregulating tumor suppressors. To date, several oncogenes, including T-cell leukemia/ lymphoma 1 (TCL1), (Mcl-1), B-Myb, CDC42, and PIK3R1, have been characterized as targets of the miR-29 family. ${ }^{33,62,63}$ Moreover, studies have showed that miR-29 family members can upregulate $\mathrm{p} 53$ levels by directly targeting p 85 and $\mathrm{CDC} 42 .{ }^{36}$

As we mentioned in te section of "role of miR-29b in epigenetic regulation", miR-29b is a crucial epi-miRNA that may revert aberrant DNA methylation by directly targeting both DNMT3A and DNMT3B. ${ }^{23,64}$ In addition, it was demonstrated that miR-29b directly targets the $3^{\prime}$ untranslated region of PDPN and inhibits invasion, apoptosis, and proliferation of glioblastomas. ${ }^{65}$ Additionally, in NSCLCs, miR-29b was identified as an upstream molecule of PTEN. miR-29b regulates PTEN gene expression through downregulation of DNMT expression and by inducing hypomethylation of the PTEN promoter, thereby exerting tumor suppressive effects. ${ }^{66}$

In MCF-7 human breast cancer cells, C1QTNF6, SPARC, and COL4A 2 were targeted by miR-29b. The degradation of any one of these mRNAs may promote invasion of MCF-7 cells. ${ }^{59}$ Notably, miR-29a was shown to directly target B7-H3, which encodes an immunomodulatory surface glycoprotein. The targeting of B7-H3 by miR-29 is implicated in immune escape by solid tumors. ${ }^{67}$

\section{Signaling pathways regulated by $\mathbf{m i R}-29 b$}

In addition to numerous cancer-related genes, signaling pathways controlling cancer activity also interact with miR-29b (Figure 2). As we mentioned above, activation of NF- $\kappa B$ signaling via ligation of TLRs repressed miR$29 \mathrm{~b}$ expression and promoter function. miR-29 expression can be also suppressed by c-Myc and hedgehog signaling, both commonly activated during the genesis of human malignancies. ${ }^{13}$ The Wnt family is a group of secreted signaling molecules that mediate a variety of cellular processes. ${ }^{68}$ The downregulation of Wnt inhibitory factor-1 (WIF-1), a major Wnt antagonist, is a common mechanism to aberrantly activate the Wnt signaling pathway. This leads to carcinogenesis in a variety of cancers. ${ }^{69,70}$ Aberrant activation of the Wnt pathway and downregulation of Wnt antagonists are common in cancer cells. Tan et al showed that WIF-1 was silenced by DNMT3A and DNMT3B, which, in turn, are suppressed by members of the miR-29 family. Consequently, forced expression of these miRNAs in lung cancer cells blocks Wnt/b-catenin signaling and inhibits tumor growth. ${ }^{28}$

In HNSCCs, a study by Kinoshita et al showed that miR-29s were downregulated, and that they directly targeted LAM-ITG signaling. Restoration of miR-29s in two HNSCC cell lines (SAS and FaDu) revealed significant inhibition 


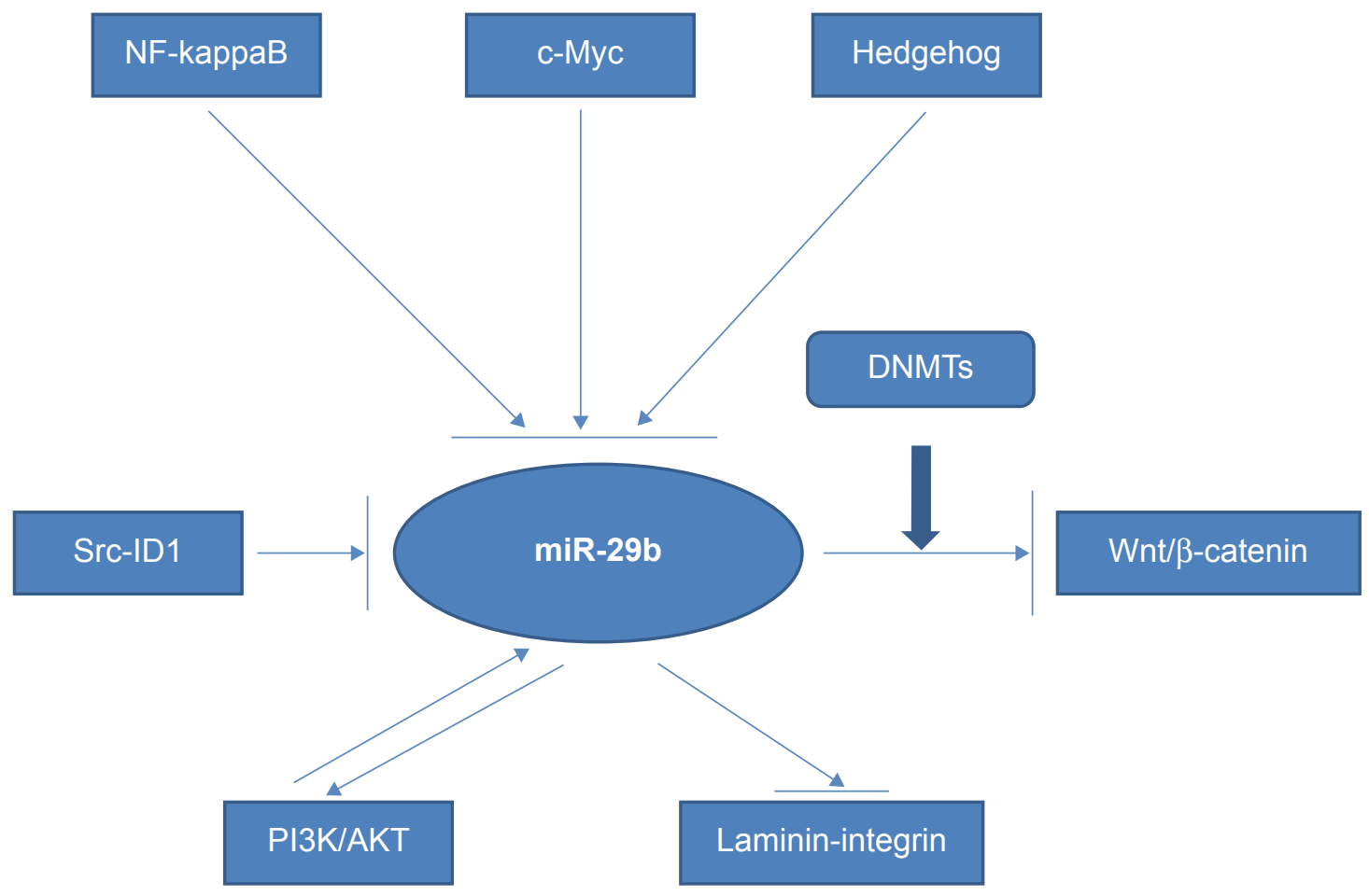

Figure 2 Interaction of the miR-29b with cellular pathways.

Abbreviations: NF-kappaB, nuclear factor kappa-light-chain-enhancer of activated B cells; c-Myc, a regulator gene that codes for a transcription factor; Src, a family of nonreceptor protein tyrosine kinases; IDI, DNA binding/differentiation I; miR-29b, micro-ribonucleic acid-29b; DNMTs, deoxyribonucleic acid transferases; Wnt, a group of secreted signaling molecules that mediate a variety of cellular processes; AKT, protein kinase $\mathrm{B}$; PI3, a kinase.

of cancer cell migration and invasion via silencing of the LAMC2 and ITGA6 genes. ${ }^{42}$

Moreover, the Src family of non-receptor protein tyrosine kinases consists of nine members. The Src signaling pathway is involved in normal cell adhesion, migration, angiogenesis, cell cycle control, and survival. The inhibitor of DNA binding/ differentiation 1 (ID1) has been identified as an important downstream mediator of Src. ${ }^{71}$ On one hand, activated c-Src kinase can repress miR-29b through b-catenin and c-Myc signaling, thereby promoting ID1 expression and invasion. On the other hand, miR-29b may directly inhibit ID1. The Src inhibitor, saracatinib, attenuates c-Myc induction and thereby increases the transcription of miR-29b. This, in turn, reduces ID1 levels and subsequent cell invasion. Consequently, a new miRNA pathway has been proposed whereby Src cooperates with the Myc pathway to induce ID1 expression by miR-29b inhibition. ${ }^{72}$ This study sheds light on the many effects of miR-29b among various signaling pathways, as well as its interaction with different signaling pathways, such as the Myc and Src-ID1 pathways. ${ }^{72}$

Finally, the regulation of the miR-29 family on cell proliferation and apoptosis is dependent on the AKT pathway and p-Rb levels. ${ }^{37}$ In MM, the PI3K/AKT pathway is a negative regulator of miR-29b. Conversely, miR-29b behaves as a negative regulator of PI3K/AKT pathway by reducing AKT phosphorylation. ${ }^{47}$

\section{Conclusion and future directions}

In summary, evidence suggests that miR-29b acts as a crucial regulator in a variety of cancers, mainly through its targeting of related genes and interactions with several signaling pathways. It can either act as a tumor suppressor, influencing epigenetic regulation, cell proliferation, apoptosis, differentiation, metastasis, and chemosensitivity, or as a tumor promoter. With regard to its controversial role in cancer, further studies with larger sample sizes are necessary. At the clinical level, a better understanding of the specific mechanisms regulating miR-29b-mediated cancer chemosensitivity will enable promising new strategies to overcome cancer chemoresistance in the future.

\section{Acknowledgment}

This study was supported by Science and Technology Agency of Hunan province in 2013 (grant number 2013FJ3055).

\section{Disclosure}

The author reports no conflicts of interest in this work. 


\section{References}

1. Bartel DP. MicroRNAs: target recognition and regulatory functions. Cell. 2009;136(2):215-233.

2. Auyeung VC, Ulitsky I, McGeary SE, Bartel DP. Beyond secondary structure: primary-sequence determinants license pri-miRNA hairpins for processing. Cell. 2013;152(4):844-858.

3. Pillai RS, Bhattacharyya SN, Filipowicz W. Repression of protein synthesis by miRNAs: how many mechanisms? Trends Cell Biol. 2007;17(3):118-126.

4. Slack FJ, Weidhaas JB. MicroRNA in cancer prognosis. $N$ Engl J Med. 2008;359(25):2720-2722.

5. Filipowicz W, Bhattacharyya SN, Sonenberg N. Mechanisms of posttranscriptional regulation by microRNAs: are the answers in sight? Nat Rev Genet. 2008;9(2):102-114.

6. Kole AJ, Swahari V, Hammond SM, Deshmukh M. miR-29b is activated during neuronal maturation and targets $\mathrm{BH} 3$-only genes to restrict apoptosis. Genes Dev. 2011;25(2):125-130.

7. Han YC, Park CY, Bhagat G, et al. microRNA-29a induces aberrant self-renewal capacity in hematopoietic progenitors, biased myeloid development, and acute myeloid leukemia. J Exp Med. 2010;207(3): 475-489.

8. Nguyen T, Kuo C, Nicholl MB, et al. Downregulation of microRNA-29c is associated with hypermethylation of tumor-related genes and disease outcome in cutaneous melanoma. Epigenetics. 2011;6(3):388-394.

9. Eyholzer M, Schmid S, Wilkens L, Mueller BU, Pabst T. The tumoursuppressive miR-29a/b1 cluster is regulated by CEBPA and blocked in human AML. Br J Cancer. 2010;103(2):275-284.

10. Chang TC, Yu D, Lee YS, et al. Widespread microRNA repression by Myc contributes to tumorigenesis. Nat Genet. 2008;40(1):43-50.

11. Zhang X, Zhao X, Fiskus W, et al. Coordinated silencing of MYCmediated miR-29 by HDAC3 and EZH2 as a therapeutic target of histone modification in aggressive B-Cell lymphomas. Cancer Cell. 2012;22(4):506-523

12. Sampath D, Liu C, Vasan K, et al. Histone deacetylases mediate the silencing of miR-15a, miR-16, and miR-29b in chronic lymphocytic leukemia. Blood. 2012;119(5):1162-1172.

13. Mott JL, Kurita S, Cazanave SC, Bronk SF, Werneburg NW, Fernandez-Zapico ME. Transcriptional suppression of mir-29b-1/mir29a promoter by c-Myc, hedgehog, and NF-kappaB. J Cell Biochem. 2010;110(5):1155-1164.

14. Winbanks CE, Wang B, Beyer C, et al. TGF-beta regulates miR-206 and miR-29 to control myogenic differentiation through regulation of HDAC4. J Biol Chem. 2011;286(16):13805-13814.

15. Wang H, Garzon R, Sun H, et al. NF-kappaB-YY1-miR-29 regulatory circuitry in skeletal myogenesis and rhabdomyosarcoma. Cancer Cell. 2008;14(5):369-381.

16. Chou J, Lin JH, Brenot A, Kim JW, Provot S, Werb Z. GATA3 suppresses metastasis and modulates the tumour microenvironment by regulating microRNA-29b expression. Nat Cell Biol. 2013;15(2):201-213.

17. Steele R, Mott JL, Ray RB. MBP-1 upregulates miR-29b that represses Mcl-1, collagens, and matrix-metalloproteinase-2 in prostate cancer cells. Genes Cancer. 2010;1(4):381-387.

18. Cittelly DM, Finlay-Schultz J, Howe EN, et al. Progestin suppression of miR-29 potentiates dedifferentiation of breast cancer cells via KLF4. Oncogene. 2013;32(20):2555-2564.

19. Schmitt MJ, Philippidou D, Reinsbach SE, et al. Interferon-gammainduced activation of Signal Transducer and Activator of Transcription 1 (STAT1) up-regulates the tumor suppressing microRNA-29 family in melanoma cells. Cell Commun Signal. 2012;10(1):41.

20. Batliner J, Buehrer E, Federzoni EA, et al. Transcriptional regulation of MIR29B by PU.1 (SPI1) and MYC during neutrophil differentiation of acute promyelocytic leukaemia cells. Br J Haematol. 2012; 157(2):270-274

21. Garzon R, Liu S, Fabbri M, et al. MicroRNA-29b induces global DNA hypomethylation and tumor suppressor gene reexpression in acute myeloid leukemia by targeting directly DNMT3A and 3B and indirectly DNMT1. Blood. 2009;113(25):6411-6418.
22. Jacobsen A, Silber J, Harinath G, Huse JT, Schultz N, Sander C. Analysis of microRNA-target interactions across diverse cancer types. Nat Struct Mol Biol. 2013;20(11):1325-1332.

23. Amodio N, Leotta M, Bellizzi D, et al. DNA-demethylating and antitumor activity of synthetic miR-29b mimics in multiple myeloma. Oncotarget. 2012;3(10):1246-1258.

24. Mishra A, Liu S, Sams GH, et al. Aberrant overexpression of IL-15 initiates large granular lymphocyte leukemia through chromosomal instability and DNA hypermethylation. Cancer Cell. 2012;22(5):645-655.

25. Amodio N, Bellizzi D, Leotta M, et al. miR-29b induces SOCS-1 expression by promoter demethylation and negatively regulates migration of multiple myeloma and endothelial cells. Cell Cycle. 2013; 12(23):3650-3662.

26. Bernot KM, Nemer JS, Santhanam R, et al. Eradicating acute myeloid leukemia in a M1l(PTD/wt):Flt3(ITD/wt) murine model: a path to novel therapeutic approaches for human disease. Blood. 2013; 122(23):3778-3783.

27. Pandey M, Sultana S, Gupta KP. Involvement of epigenetics and microRNA-29b in the urethane induced inception and establishment of mouse lung tumors. Exp Mol Pathol. 2014;96(1):61-70.

28. Tan M, Wu J, Cai Y. Suppression of Wnt signaling by the miR-29 family is mediated by demethylation of WIF-1 in non-small-cell lung cancer. Biochem Biophys Res Commun. 2013;438(4):673-679.

29. Galli R, Paone A, Fabbri M, et al. Toll-like receptor 3 (TLR3) activation induces microRNA-dependent reexpression of functional RARbeta and tumor regression. Proc Natl Acad Sci U S A. 2013;110(24): 9812-9817.

30. Morita S, Horii T, Kimura M, Ochiya T, Tajima S, Hatada I. miR-29 represses the activities of DNA methyltransferases and DNA demethylases. Int J Mol Sci. 2013;14(7):14647-14658.

31. Mott JL, Kobayashi S, Bronk SF, Gores GJ. mir-29 regulates Mcl-1 protein expression and apoptosis. Oncogene. 2007;26(42):6133-6140.

32. Aldaz B, Sagardoy A, Nogueira L, et al. Involvement of miRNAs in the differentiation of human glioblastoma multiforme stem-like cells PLoS One. 2013;8(10):e77098.

33. Garzon R, Heaphy CE, Havelange V, et al. MicroRNA 29b functions in acute myeloid leukemia. Blood. 2009;114(26):5331-5341.

34. Xiong Y, Fang JH, Yun JP, et al. Effects of microRNA-29 on apoptosis, tumorigenicity, and prognosis of hepatocellular carcinoma. Hepatology. 2010;51(3):836-845.

35. Zhang YK, Wang H, Leng Y, et al. Overexpression of microRNA-29b induces apoptosis of multiple myeloma cells through down regulating Mcl-1. Biochem Biophys Res Commun. 2011;414(1):233-239.

36. Park SY, Lee JH, Ha M, Nam JW, Kim VN. miR-29 miRNAs activate p53 by targeting p85 alpha and CDC42. Nat Struct Mol Biol. 2009;16(1): 23-29.

37. Gong JN, Yu J, Lin HS, et al. The role, mechanism and potentially therapeutic application of microRNA-29 family in acute myeloid leukemia. Cell Death Differ. 2014;21(1):100-112.

38. Rossi M, Pitari MR, Amodio N, et al. miR-29b negatively regulates human osteoclastic cell differentiation and function: implications for the treatment of multiple myeloma-related bone disease. J Cell Physiol. 2013;228(7):1506-1515.

39. Melo SA, Kalluri R. miR-29b moulds the tumour microenvironment to repress metastasis. Nat Cell Biol. 2013;15(2):139-140.

40. Ru P, Steele R, Newhall P, Phillips NJ, Toth K, Ray RB. miRNA-29b suppresses prostate cancer metastasis by regulating epithelial-mesenchymal transition signaling. Mol Cancer Ther. 2012;11(5):1166-1173.

41. Poudyal D, Cui X, Le PM, et al. A key role of microRNA-29b for the suppression of colon cancer cell migration by American ginseng. PLoS One. 2013;8(10):e75034.

42. Kinoshita T, Nohata N, Hanazawa T, et al. Tumour-suppressive microRNA-29s inhibit cancer cell migration and invasion by targeting laminin-integrin signalling in head and neck squamous cell carcinoma. Br J Cancer. 2013;109(10):2636-2645.

43. Dai Z, Huang Y, Sadee W. Growth factor signaling and resistance to cancer chemotherapy. Curr Top Med Chem. 2004;4(13):1347-1356. 
44. Meng F, Henson R, Lang M, et al. Involvement of human micro-RNA in growth and response to chemotherapy in human cholangiocarcinoma cell lines. Gastroenterology. 2006;130(7):2113-2129.

45. Okamoto K, Miyoshi K, Murawaki Y. miR-29b, miR-205 and miR-221 enhance chemosensitivity to gemcitabine in $\mathrm{HuH} 28$ human cholangiocarcinoma cells. PLoS One. 2013;8(10):e77623.

46. Dai F, Zhang Y, Zhu X, Shan N, Chen Y. The anti-chemoresistant effect and mechanism of MUC1 aptamer-miR-29b chimera in ovarian cancer. Gynecol Oncol. 2013;131(2):451-459.

47. Amodio N, Di Martino MT, Foresta U, et al. miR-29b sensitizes multiple myeloma cells to bortezomib-induced apoptosis through the activation of a feedback loop with the transcription factor Sp1. Cell Death Dis. 2012;3:e436.

48. Mims A, Walker AR, Huang X, et al. Increased anti-leukemic activity of decitabine via AR-42-induced upregulation of miR-29b: a novel epigenetic-targeting approach in acute myeloid leukemia. Leukemia. 2013;27(4):871-878.

49. Blum W, Garzon R, Klisovic RB, et al. Clinical response and miR-29b predictive significance in older AML patients treated with a 10-day schedule of decitabine. Proc Natl Acad Sci U S A. 2010;107(16):7473-7478.

50. Blum W, Schwind S, Tarighat SS, et al. Clinical and pharmacodynamic activity of bortezomib and decitabine in acute myeloid leukemia. Blood. 2012;119(25):6025-6031.

51. Huang X, Schwind S, Yu B, et al. Targeted delivery of microRNA-29b by transferrin-conjugated anionic lipopolyplex nanoparticles: a novel therapeutic strategy in acute myeloid leukemia. Clin Cancer Res. 2013;19(9):2355-2367.

52. Wu Y, Crawford M, Mao Y, et al. Therapeutic delivery of microRNA$29 \mathrm{~b}$ by cationic lipoplexes for lung cancer. Mol Ther Nucleic Acids. 2013;2:e84

53. Chen $\mathrm{CZ}$. MicroRNAs as oncogenes and tumor suppressors. $N$ Engl J Med. 2005;353(17):1768-1771.

54. Santanam U, Zanesi N, Efanov A, et al. Chronic lymphocytic leukemia modeled in mouse by targeted miR-29 expression. Proc Natl Acad Sci US A. 2010;107(27):12210-12215.

55. Kong G, Zhang J, Zhang S, Shan C, Ye L, Zhang X. Upregulated microRNA-29a by hepatitis B virus X protein enhances hepatoma cell migration by targeting PTEN in cell culture model. PLoS One. 2011; 6(5):e19518.

56. Cheng J, Guo S, Chen S, et al. An extensive network of TET2targeting MicroRNAs regulates malignant hematopoiesis. Cell Rep. 2013;5(2):471-481.

57. Xu F, Zhang Q, Cheng W, Zhang Z, Wang J, Ge J. Effect of miR-29b-1* and miR-29c knockdown on cell growth of the bladder cancer cell line T24. J Int Med Res. 2013;41(6):1803-1810.
58. Wang C, Bian Z, Wei D, Zhang JG. miR-29b regulates migration of human breast cancer cells. Mol Cell Biochem. 2011;352(1-2):197-207.

59. Wang C, Gao C, Zhuang JL, Ding C, Wang Y. A combined approach identifies three mRNAs that are down-regulated by microRNA-29b and promote invasion ability in the breast cancer cell line MCF-7. J Cancer Res Clin Oncol. 2012;138(12):2127-2136.

60. Fu J, Tang W, Du P, et al. Identifying microRNA-mRNA regulatory network in colorectal cancer by a combination of expression profile and bioinformatics analysis. BMC Syst Biol. 2012;6:68.

61. Luna C, Li G, Qiu J, Epstein DL, Gonzalez P. Role of miR-29b on the regulation of the extracellular matrix in human trabecular meshwork cells under chronic oxidative stress. Mol Vis. 2009;15:2488-2497.

62. Pekarsky Y, Santanam U, Cimmino A, et al. Tcl1 expression in chronic lymphocytic leukemia is regulated by miR-29 and miR-181. Cancer Res. 2006;66(24):11590-11593.

63. Martinez I, Cazalla D, Almstead LL, Steitz JA, DiMaio D. miR-29 and miR-30 regulate B-Myb expression during cellular senescence. Proc Natl Acad Sci U S A. 2011;108(2):522-527.

64. Fabbri M, Garzon R, Cimmino A, et al. MicroRNA-29 family reverts aberrant methylation in lung cancer by targeting DNA methyltransferases 3A and 3B. Proc Natl Acad Sci U S A. 2007;104(40):15805-15810.

65. Cortez MA, Nicoloso MS, Shimizu M, et al. miR-29b and miR-125a regulate podoplanin and suppress invasion in glioblastoma. Genes Chromosomes Cancer. 2010;49(11):981-990.

66. Li G, Zhao J, Peng X, Liang J, Deng X, Chen Y. The mechanism involved in the loss of PTEN expression in NSCLC tumor cells. Biochem Biophys Res Commun. 2012;418(3):547-552.

67. Xu H, Cheung IY, Guo HF, Cheung NK. MicroRNA miR-29 modulates expression of immunoinhibitory molecule B7-H3: potential implications for immune based therapy of human solid tumors. Cancer Res. 2009;69(15):6275-6281.

68. MacDonald BT, Tamai K, He X. Wnt/beta-catenin signaling: components, mechanisms, and diseases. Dev Cell. 2009;17(1):9-26.

69. Lin YC, You L, Xu Z, et al. Wnt signaling activation and WIF-1 silencing in nasopharyngeal cancer cell lines. Biochem Biophys Res Commun. 2006;341(2):635-640.

70. Mazieres J, He B, You L, et al. Wnt inhibitory factor-1 is silenced by promoter hypermethylation in human lung cancer. Cancer Res. 2004; 64(14):4717-4720.

71. Gautschi O, Tepper CG, Purnell PR, et al. Regulation of Id1 expression by SRC: implications for targeting of the bone morphogenetic protein pathway in cancer. Cancer Res. 2008;68(7):2250-2258.

72. Rothschild SI, Tschan MP, Federzoni EA, et al. MicroRNA-29b is involved in the Src-ID1 signaling pathway and is dysregulated in human lung adenocarcinoma. Oncogene. 2012;31(38):4221-4232.
OncoTargets and Therapy

\section{Publish your work in this journal}

OncoTargets and Therapy is an international, peer-reviewed, open access journal focusing on the pathological basis of all cancers, potential targets for therapy and treatment protocols employed to improve the management of cancer patients. The journal also focuses on the impact of management programs and new therapeutic agents and protocols on
Dovepress

patient perspectives such as quality of life, adherence and satisfaction The manuscript management system is completely online and includes a very quick and fair peer-review system, which is all easy to use. Visit http://www.dovepress.com/testimonials.php to read real quotes from published authors. 